\title{
The Peculiar Object IRAS 06088+1909
}

\author{
A. RICHICHI ${ }^{1}$, G. CALAMAI ${ }^{1}$, F. LISI $^{1}$, \\ B. STECKLUM ${ }^{2}$, T. HERBST ${ }^{3}$ and E. THAMM ${ }^{4}$
}

1 Arcetri Observatory, Florence, Italy

2 Thüringer Landessternwarte, Tautenburg, Germany

${ }^{3}$ Max-Planck Institut für Astronomie, Heidelberg, Germany

${ }^{4}$ Max-Planck Research Unit "Dust in Star Forming Regions" Jena, Germany

Our observation of IRAS $06088+1909$ began with two occultation events recorded in the $K$ band $(2.2 \mu \mathrm{m})$ during two simultaneous runs at the CAHA, Calar Alto, and TIRGO observatories in October 1994. The difference in latitude of these two sites insures a sufficiently different scan angle to investigate deviations from spherical symmetry. These first occultations were followed by broad-band photometry in the $1-5 \mu \mathrm{m}$ range obtained at TIRGO. The interesting characteristics of this star stimulated further observations: (a) imaging and polarimetry in the $V, R, I$ bands; (b) IR speckle interferometry; $(c)$ near-IR low resolution spectroscopy; and $(d)$ near-IR polarimetry. The sequence of observations was concluded by observing the occultation of January 1995, again from both CAHA ( $K$ filter) and TIRGO ( $L$ filter at 3.8 $\mu \mathrm{m})$. Of the four lunar occultations, only the Calar Alto light curve from October 1994 is consistent with a circular stellar disk. In this case, however, the diameter of $00^{\prime \prime} 007$ would imply a very low photospheric temperature $\left(T_{\text {eff }}<1500 \mathrm{~K}\right)$. In all the remaining light curves, the data show that more extended emission is present. This is particularly evident in the $L$-band light curve. Photometric results show that the star is extremely heavily reddened $(V-K \approx 14 \mathrm{mag})$; this amount of extinction cannot be ascribed to interstellar dust and must be produced locally, presumably in a circumstellar shell. The polarization measurements indicate that there might be a departure from spherical symmetry in the shell. The $K$ band spectrum also indicates that the star is very red, but more importantly, it shows evidence of at least some photospheric lines, indicating that the shell is not completely optically thick at these wavelengths. Speckle interferometry shows the source to be completely unresolved at the diffraction limit of the telescope in the $K$ filter $(0.13)$. The evidence for extended emission around IRAS $06088+1909$ is incontrovertible, and our results indicate some resemblance to the case of IRC +10216. IRAS $06088+1909$ is probably of a less extreme nature, but it is nevertheless a quite peculiar object that will deserve further investigation. 